\title{
Obtención del índice de fallas de líneas de distribución ante impactos indirectos de rayo en zona tropical
}

\section{Calculation of indirect-lightning flashover rate on distribution lines from indirect lightning in tropical zones}

\author{
Edison Soto-Ríos ${ }^{1}$, Ernesto Pérez-González ${ }^{2}$, Diego del Río-Trujillo ${ }^{3}$ \\ ${ }^{1}$ Grupo de Investigación en Sistemas de Energía Eléctrica-GISEL, Escuela de Ingeniería Eléctrica, Electrónica y \\ Telecomunicaciones, Universidad Industrial de Santander, Bucaramanga, Colombia. Email: easotor@uis.edu.co \\ ${ }^{2}$ Programa de investigación sobre adquisición y análisis de señales-PAAS. Departamento de Energía Eléctrica y Automática, \\ Universidad Nacional de Colombia, Medellín, Colombia. Email: Email: eperezg@unal.edu.co \\ ${ }^{3}$ Departamento de Ingeniería, Eléctrica, Electrónica y Computación, Universidad Nacional de Colombia - Manizales, Colombia. \\ Email: dfdelriot@unal.edu.co
}

RECIBIDO: Abril 20, 2017. ACEPTADO: Julio 19, 2017. VERSIÓN FINAL: Octubre 22, 2017.

\section{RESUMEN}

En este artículo se obtiene el índice de fallas por impactos indirectos de una línea de distribución siguiendo el procedimiento descrito en el estándar IEEE 1410 de 2010, pero cambiando los parámetros de las distribuciones de la primera descarga de retorno (corriente pico y tiempo de frente) adoptados por CIGRÉ por los obtenidos de 38 mediciones de corrientes en la estación experimental del Morro do Cachimbo en Belo Horizonte - Brasil, que son representativas de la zona tropical. Se encuentra como resultado que la tasa de fallas calculada con los datos de zona tropical es mayor a los que se obtienen con las curvas del CIGRÉ. Dichos incrementos pueden ser en el caso más extremo de hasta $70 \%$.

PALABRAS CLAVE: Parámetros del rayo; índice de fallas; líneas de distribución; tensiones inducidas; CIGRÉ; MCS.

\begin{abstract}
In this paper the indirect-lightning flashover rate of a distribution line is obtained following the procedure described in the IEEE Std. 1410 - 2010, but using other parameters of the first stroke distributions (peak current and front time) adopted by CIGRÉ. These parameters were taken from the measured currents of the Morro do Cachimbo Station in Belo Horizonte - Brazil, which are representative of the tropical zone. As a result, the calculated flashover rate with the data of tropical zone is higher than one obtained with the CIGRÉ curves. Such increments could be in the most extreme case of $70 \%$.
\end{abstract}

KEYWORDS: Lightning parameters; flashover rate; distribution lines; lightning induced voltages; CIGRÉ; MCS.

ISSN Impreso: 1657 - 4583, En Línea: 2145 - 8456

Este artículo puede compartirse bajo la licencia CC BY-ND 4.0 y se referencia usando el siguiente formato: E. Soto, E. Pérez, D. del Río, "Obtención del índice de fallas de líneas de distribución ante impactos indirectos de rayo en zona tropical,” Rev. UIS Ing., vol. 17, no. 1, pp. 217-222, 2018. Doi: https://doi.org/10.18273/revuin.v17n1-2018021 


\section{INTRODUCCIÓN}

Los parámetros del rayo a nivel mundial se construyeron basados en la medición de corrientes sobre torres elevadas. Con base en un total de 338 descargas negativas a tierra, obtenidas en diferentes partes del mundo y diferentes estructuras (76 rayos en líneas y 262 en mástiles y torres) de diferentes alturas, generalmente menores a $60 \mathrm{~m}$ [1], el CIGRÉ adoptó una distribución de probabilidad para la corriente pico de la primera descarga de retorno del tipo log-normal dividida en dos distribuciones, cada una con media $\mu_{I}$ y desviación estándar logarítmica $\sigma_{I}$, dada por la siguiente expresión:

$f(I)=\frac{1}{\sqrt{2 \pi} \sigma_{I} I} e^{-\frac{\left(\ln \frac{I}{\mu_{I}}\right)^{2}}{2 \sigma_{I}^{2}}}$
Los parámetros de las dos sub-distribuciones de probabilidad se presentan en la Tabla 1 [4].

Tabla 1. Parámetros de la Distribución de Probabilidad Log normal para la primera descarga de retorno en descargas negativas adoptados por CIGRÉ [4].

\begin{tabular}{|c|c|c|c|c|}
\hline Parámetro & $\begin{array}{r}\mathrm{M} \\
I_{p} \leq 20 k\end{array}$ & $\begin{array}{l}> \\
>20 \mathrm{kA}\end{array}$ & $\begin{array}{r}\text { Des } \\
\text { es } \\
I_{p} \leq 20 k\end{array}$ & $20 \mathrm{kA}$ \\
\hline$I_{p}(\mathrm{kA})$ & $61 \mathrm{kA}$ & $33.3 \mathrm{kA}$ & 1.33 & 0.605 \\
\hline$t_{f}(\mu \mathrm{s})$ & \multicolumn{2}{|c|}{$3.83 \mu \mathrm{s}$} & \multicolumn{2}{|c|}{0.553} \\
\hline
\end{tabular}

En los países ubicados en zona tropical se han construido curvas de distribución de probabilidad de corrientes con base en mediciones directas e indirectas de rayos como se muestra en la

Figura 1.

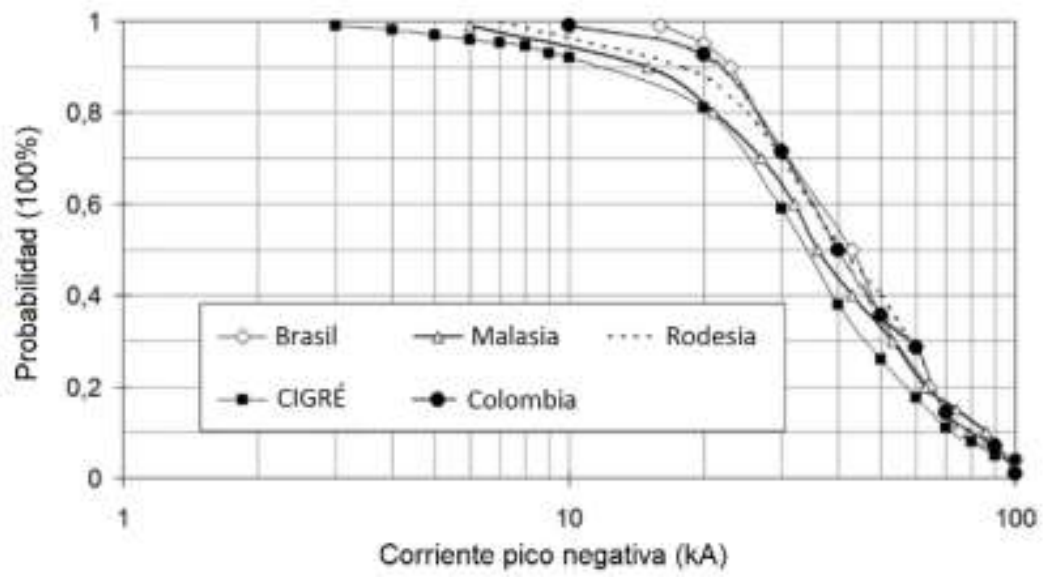

Figura 1. Distribución de probabilidad de corriente de rayo para países en zona tropical. Adaptado de [5].

En general, los valores de corriente obtenidos en cada país superan los adoptados por el CIGRÉ. En el caso de Brasil la media de la corriente fue de $45 \mathrm{kA}$ y en Colombia de $42 \mathrm{kA}$ [2][3]. Dichos resultados han sido controversiales respecto a los obtenidos en zonas templadas en los que la media de $30 \mathrm{kA}$ ha sido consistente. Las diferencias de los parámetros del rayo para zona tropical ha sido discutida en diferentes artículos [5][6][7]. En [8] se analiza el efecto de las corrientes en zona tropical en el cálculo de la tasa de fallas por flameo inverso en líneas de transmisión, encontrándose importantes incrementos respecto los encontrados usando las curvas de CIGRÉ.

Hasta ahora ningún trabajo ha abordado la influencia de las distribuciones de probabilidad de zona tropical en el cálculo del índice de fallas por impactos indirectos en líneas de distribución, por tanto, en este artículo se trabajará está temática. Para esto se usarán las

mediciones más completas de corrientes para zona tropical que han sido las realizadas en Belo Horizonte Brasil [9] con un total de 38 mediciones directas. La curva de distribución de probabilidad de corriente y tiempos de frente obtenidas en [9] se usará en el procedimiento de Monte Carlo descrito en el estándar IEEE 1410-2010 [10] para obtener la tasa de fallas por impactos indirectos de rayos en una línea de distribución típica sobre terrenos planos con conductividades infinita y finita. Se realizará una comparación de los resultados obtenidos con los encontrados en el estándar IEEE 1410 -2010 en la cual se usaron los parámetros del rayo tradicionales para zonas templadas. 


\section{CÁlCUlo de LA TASA DE FALLAS DE LÍNEAS DE DISTRIBUCIÓN SEGÚN EL ESTÁNDAR IEEE 1410-2010}

La estimación del índice de fallas de una línea de distribución parte en un método propuesto por Borghetti et al [11], y adoptado por el estándar IEEE 1410 -2010 [10]. Éste se basa en el método de Monte Carlo y el uso de un software que permita calcular la tensión inducida. Para el caso de este artículo se usará el software Yaluk Draw [12], desarrollado por dos de los autores de este trabajo y propiedad de la Universidad Nacional de Colombia. La metodología de cálculo de la tasa de fallas tiene los siguientes pasos:

Se simula un largo número $\mathrm{n}_{\text {tot }}$ de impactos generados aleatoriamente, caracterizados por tres parámetros: la corriente pico $\mathrm{I}_{\mathrm{p}}$, el tiempo de frente $\mathrm{t}_{\mathrm{f}} \mathrm{y}$ la distancia a la línea $y$. Los parámetros $\mathrm{I}_{\mathrm{p}} \mathrm{y} \mathrm{t}_{\mathrm{f}}$ siguen una distribución de probabilidad log-normal adoptada por CIGRE (Tabla 1). El tiempo de frente $t_{f}$ es obtenido por medio de la corriente con un coeficiente de correlación de 0.47. El Yaluk Draw implementa como en el estándar IEEE 1410 una onda tipo rampa-plana. La distancia $y$ sigue una probabilidad uniforme de 0 a $y_{\max }$. El valor de $y_{\max }$ se escoge cuidadosamente para tener en cuenta todos los impactos indirectos que exceden el CFO de la línea de distribución.
Los impactos que caen muy cerca de la línea son eliminados por ser impactos directos de acuerdo con el método de incidencia electrogeométrico de Whitehead [10]. Para cada impacto indirecto la máxima tensión inducida es calculada por medio del Yaluk Draw. Con $n$ el número de eventos que generan tensiones inducidas mayores que el nivel de aislamiento (el $C F O$ multiplicado por un factor de 1.5) y $\mathrm{Ng}$ la densidad de descargas a tierra anuales, el índice de fallas por $100 \mathrm{~km}$ de las líneas de distribución $\left(\mathrm{F}_{\mathrm{p}}\right)$ se calcula como:

$F_{p}=200 \frac{n}{n_{\text {tot }}} N_{g} y_{\max }$

De acuerdo con la metodología anterior, Borguetti [11] calculan la tasa de fallas por impactos indirectos de una línea de distribución típica para diferentes conductividades del terreno. Esta se adopta en el estándar IEEE 1410 - 2010 [10]. Dicho resultado se muestra en la

Figura 2. Se puede ver que la conductividad del terreno tiene un efecto importante en el incremento del número de flameos en la línea. Según este resultado, una línea con un CFO de $300 \mathrm{kV}$ ubicada sobre un terreno con conductividad infinita no presentaría fallas por flameos ante impactos indirectos [10]. Si la conductividad es de $1 \mathrm{mS} / \mathrm{m}$, el CFO debe ser de $420 \mathrm{kV}$.

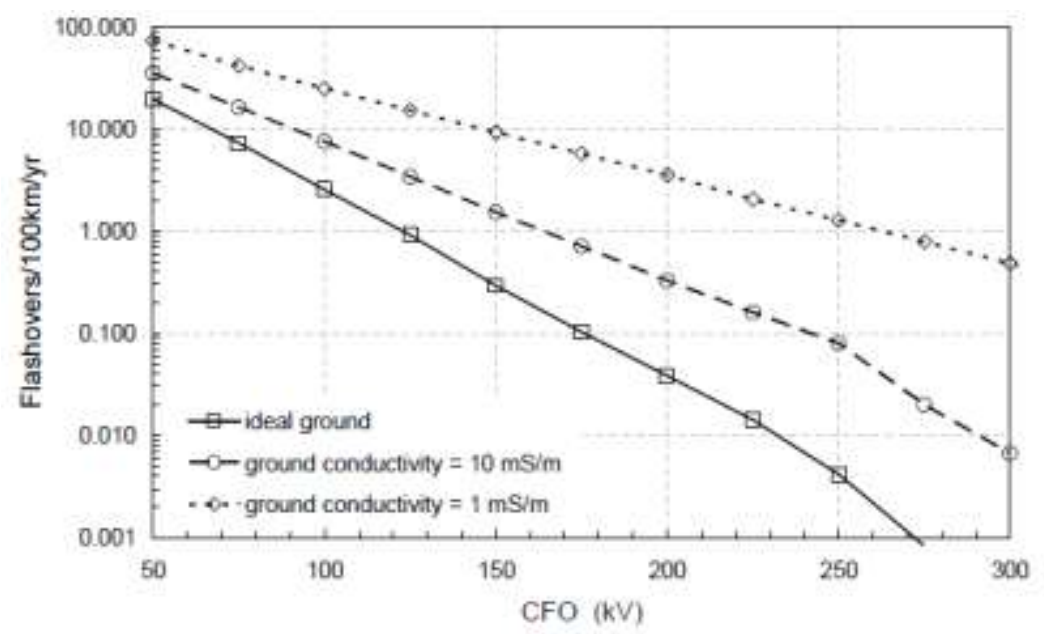

Figura 2. Número de flameos por tensiones inducidas vs el nivel de aislamiento de acuerdo al estándar IEEE 1410. Tomado de [10].

\section{CÁlCulo de la tasa de FAllas POR IMPACTOS INDIRECTOS EN ZONA TROPICAL}

Los parámetros del rayo obtenidos en zona tropical difieren de los encontrados en zonas templadas. La mayor cantidad de mediciones de corrientes de rayos en zona tropical son las encontrados en el Morro do Cachimbo (MCS, por sus siglas en inglés) en Belo Horizonte - Brasil, con un total de 38 mediciones de la corriente pico de la primera descarga de retorno, hasta 2012 [9][13]. En la Tabla 2 se presentan los parámetros estadísticos de la distribución log-normal para la 
corriente pico $\mathrm{I}_{\mathrm{p}}$ y el tiempo de frente $\mathrm{t}_{\mathrm{f}}$ obtenidos de las mediciones del Morro do Cachimbo (MCS). Se puede notar un valor considerablemente mayor al medido en zona templadas en las que la mediana fue de $31 \mathrm{kA}$. En la Figura 3 se presentan la comparación de las curvas de distribución de corriente obtenida en el Morro do Cachimbo (MCS) y la distribución adoptada por CIGRÉ.

Se puede ver en general probabilidades mayores de encontrar corrientes entre 0 a $100 \mathrm{kA}$ para la distribución MCS que para el CIGRÉ. Más allá de 100 kA la curva MCS disminuye respecto a la curva de CIGRÉ, posiblemente debido al poco número de mediciones realizadas en MCS mayores a 100 kA (sólo una) [13].

Tabla 2. Corriente pico y tiempo de frente para zona tropical de acuerdo a [9] y [13].

\begin{tabular}{|c|c|c|}
\hline Parámetro & Mediana & Desviación estándar \\
\hline$I_{p}(\mathrm{kA})$ & 45 & 0.45 \\
\hline$t_{f}(\mu \mathrm{s})$ & 4.3 & 0.62 \\
\hline
\end{tabular}

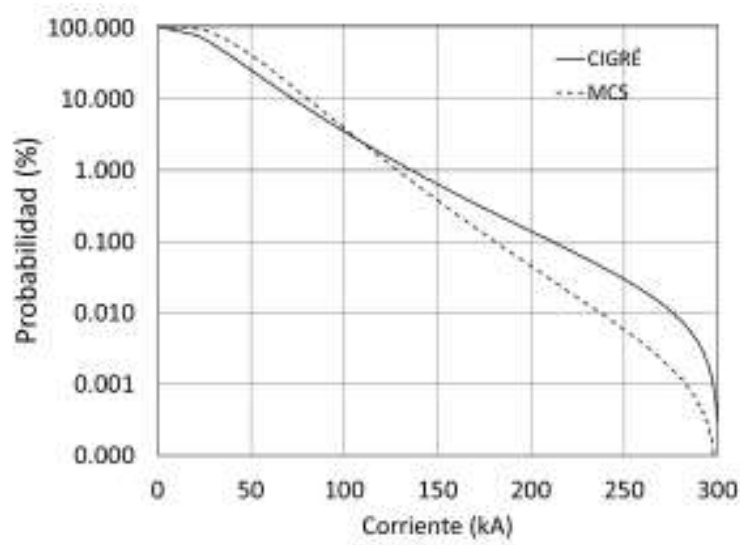

Figura 3. Comparación de las distribuciones de probabilidad de corriente de corriente de CIGRÉ y medidas en la estación del Morro do Cachimbo (MCS).

Los parámetros de la curva de distribución de corrientes obtenida en MCS, descritos en la Tabla 2 se usaron en el procedimiento general de la IEEE 1410-2010, descrito en la sección 2, con el fin de obtener la tasa de fallas de una línea de distribución con las mismas características de la simulada en el mismo estándar [10]. La línea simulada fue de $2 \mathrm{~km}$ de longitud, $10 \mathrm{~m}$ de altura con un radio de $5 \mathrm{~mm}$, conectada en ambos extremos a su impedancia característica. Para describir la variación de la corriente del rayo a lo largo del canal de la descarga se usó un modelo TL y una velocidad de la descarga de retorno de $120 \mathrm{~m} / \mu \mathrm{s}$.

La tasa de fallas obtenida considerando un terreno de conductividad infinita se presenta en la Figura 4 . Se puede ver que la tasa de fallas obtenida considerando la distribución MCS es superior a la obtenida con los datos de CIGRÉ para CFOs de la línea entre 50 y $100 \mathrm{kV}$. Alcanzándose un incremento de un $40 \%$ aproximadamente. Para valores de CFO superiores a 100 $\mathrm{kV}$ la tasa de fallas desciende posiblemente como resultado del comportamiento de la curva MCS (Figura 3) después de $100 \mathrm{kA}$.

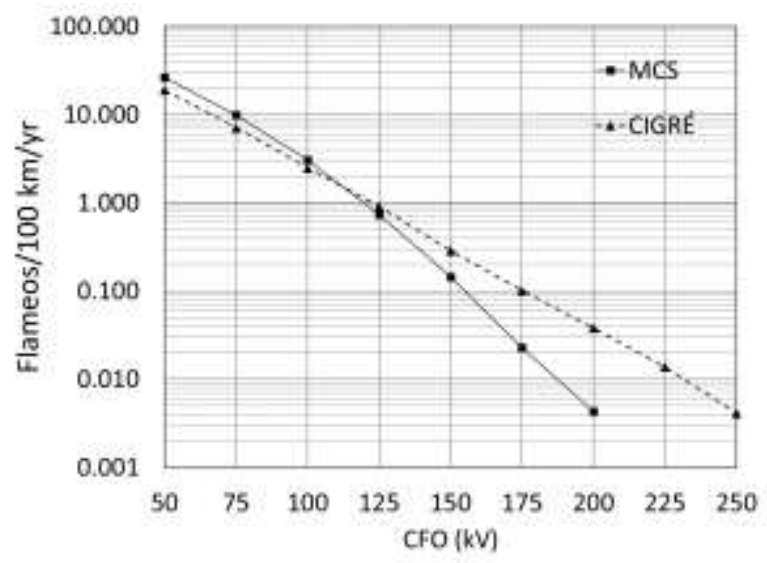

Figura 4. Tasa de fallas de una línea de distribución típica sobre un terreno perfectamente conductor simulando la distribución de corrientes obtenida en MCS, presentada en la Tabla 2.

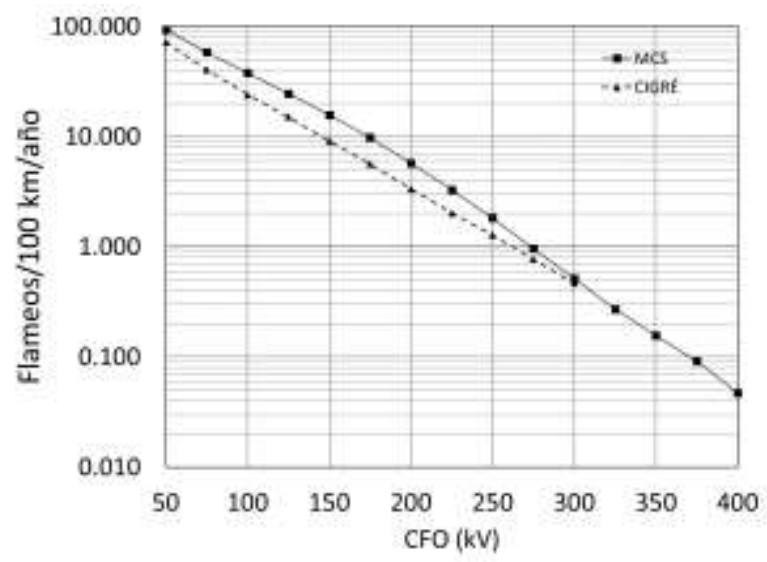

Figura 5. Tasa de fallas de una línea de distribución típica sobre un terreno con conductividad $\sigma=0.001 \mathrm{~S} / \mathrm{m}$ simulando la distribución de corrientes obtenida en MCS, presentada en la Tabla 2.

En la Figura 5, se presenta la tasa de fallas para la línea anterior ubicada ahora sobre un terreno de conductividad finita $(\sigma=1 \mathrm{mS} / \mathrm{m})$. La tasa de fallas obtenida es considerablemente superior a la encontrada para un terreno perfectamente conductor. Respecto a la obtenida tomando los datos de CIGRÉ, la curva MCS llega a ser entre 60 y $70 \%$ mayor, para CFOs menores a $200 \mathrm{kV}$. 
Para valores por encima de éste, las dos curvas tienden a acercarse, hasta que eventualmente en $300 \mathrm{kV}$ poseen valores casi iguales.

\section{CONCLUSIONES}

En este trabajo se logra incluir por primera vez el efecto de los parámetros del rayo medidos en zona tropical en el cálculo de tasas de fallas de líneas de distribución sobre suelos con y sin pérdidas. Se hizo una comparación con los únicos resultados disponibles hasta el momento que son los obtenidos en el estándar IEEE 1410 que usan las curvas del CIGRÉ.

Para conductividad infinita, se encontró que el número de flameos por $100 \mathrm{~km}$-año que presenta la línea cuando se consideran los parámetros de MCS son superiores a los de CIGRÉ para niveles de aislamiento bajos (hasta 100 $\mathrm{kV}$ ) y tienden a ser menores para niveles de aislamiento altos. Esto, siguiendo aproximadamente la tendencia de las dos curvas de probabilidad de corriente. Para conductividad finita hay un mayor número de flameos cuando se considera los parámetros de MCS, aun para valores de CFO mayores a $100 \mathrm{kV}$, esto en parte como resultado del incremento de los valores de las tensiones inducidas al incluir una conductividad baja.

Es importante a futuro construir nuevamente las curvas presentadas en este artículo con base en la información actualizada de las corrientes medidas en la estación del Morro do Cachimbo, o de mediciones realizadas en Colombia. Esto permitirá tener mayor certeza de la tasa de fallas para CFOs altos. Adicionalmente se podrá estimar con mayor exactitud el nivel de CFO mínimo que deben tener las líneas de distribución ubicadas en zona tropical para ser protegidas al efecto de las tensiones inducidas por rayos.

\section{REFERENCIAS}

[1] C. Alberto Nucci, "A survey on CIGRÉ and IEEE procedures for the estimation of the lightning performance of overhead transmission and distribution lines," 2010 Asia-Pacific International Symposium on Electromagnetic Compatibility, Beijing, 2010, pp. 11241133.

[2] ICONTEC, NTC 4552-1 Protección contra Descargas Eléctricas Atmosféricas (Rayos). Parte 1. Principios Generales. 2008.

[3] H. Torres, El Rayo. Mitos, Leyendas Ciencia y Tecnología. Bogotá, Colombia: Unibiblos, 2002.
[4] Cigré Working Group 01 of SC 33, Guide to Procedures for Estimating the Lightning Performance of Transmission Lines Cigré, no. 63, Oct., 1991.

[5] H. Torres, E. Perez, C. Younes, D. Aranguren, J. Montaña and J. Herrera, "Contribution to Lightning Parameters Study Based on Some American Tropical Regions Observations," in IEEE Journal of Selected Topics in Applied Earth Observations and Remote Sensing, vol. 8, no. 8, pp. 4086-4093, Aug., 2015.

[6] H. Torres. "Variations of Lightning Parameter Magnitudes within Space and Time". International Conference on Lightning Protection (ICLP), 1998.

[7] V. Rakov. "Lightning parameters of engineering interest: Application of lightning detection technologies". Presentation. EGAT, Bangkok, Thailand, Nov., 2012.

[8] F. Silveira and S. Visacro. The Impact of Peak Current Distribution on the Calculation of Backflashover Rate of Transmission Lines. SICEL 2015.

[9] Visacro, S. Statistical analysis of lightning current parameters: Measurements at Morro do Cachimbo Station. Journal of Geophysical Research, 109(D1), D01105. 2004. https://doi.org/10.1029/2003JD003662.

[10]Power, I., \& Society, IEEE Std 1410 - 2010. IEEE Guide for Improving the Lightning Performance of Electric Power Overhead Distribution Lines. vol. 2010. 2011.

[11] Borghetti, A., Nucci, C. A., \& Paolone, M. An Improved Procedure for the Assessment of Overhead Line Indirect Lightning Performance and Its Comparison with the IEEE Std. 1410 Method, vol. 22, no. 1, pp. 684692. 2007.

[12] Pérez, E., \& Soto, E. Software. Yaluk Draw. Universidad Nacional - Sede Manizales. 2010.

[13] Visacro, S., Mesquita, C. R., De Conti, A., \& Silveira, F. H. Updated statistics of lightning currents measured at Morro do Cachimbo Station. Atmospheric Research, vol. 117, pp. 55-63. 2012. https://doi.org/10.1016/j.atmosres.2011.07.010.

[14] H. Rojas, A. Cruz, C. Cortez, "Características de los campos eléctricos generados por rayos medidos en la Sabana de Bogotá, Colombia," Rev. UIS Ing., vol. 16, no. 2, pp. $243-252,2017$.

[15] A. Vanegas, E. Velilla, A. Valencia, "Evaluación del desempeño eléctrico de estructuras de diferentes materiales empleadas en redes de distribución," Rev.UIS Ing., vol. 17, no. 1, pp. 35-42, 2018.

[16]V. Barrera-Núñez, G. Carrillo-Caicedo, G. OrdoñezPlata, J. Mora-Flórez, "Una aplicación de la técnica 


\section{RNIIS REVISTA UIS}

LAMDA a los índices de continuidad del suministro de energía eléctrica." Rev.UIS Ing., vol. 5, no. 1, pp. 25-36, 2006. 